\title{
Heterosis and Combining Ability in CMS Based Hybrid Chilli (Capsicum annuum L.)
}

\author{
B. V. Tembhurne ${ }^{1} \&$ S. K. Rao ${ }^{1,2}$ \\ ${ }^{1}$ Department of Plant Breeding and Genetics, Jawaharlal Nehru Krishi Vishwa Vidyalaya, Jabalpur (JNKVV), \\ Madhya Pradesh, India \\ ${ }^{2}$ Director Farms, Jawaharlal Nehru Krishi Vishwa Vidyalaya, Jabalpur, Madhya Pradesh, India \\ Correspondence: B. V. Tembhurne, College of Agriculture, University of Agricultural Sciences, Raichur 584 101, \\ Karnataka, India. E-mail: bvtembhurne@gmail.com
}

Received: May 30, 2012 Accepted: June 16, 2012 Online Published: August 24, 2012

doi:10.5539/jas.v4n10p89 URL: http://dx.doi.org/10.5539/jas.v4n10p89

\begin{abstract}
Field experiments were conducted during kharif 2009-2010 to identify the most heterotic CMS based hybrids in chilli. The heterosis for dry fruit yield and yield components of $51 \mathrm{~F}_{1}$ hybrids were derived from crosses between three females (line) and seventeen males (tester) crossed in line $\mathrm{x}$ tester fashion. The cross ACA1/LCA334 exhibited significant heterosis over mid parent $493.44 \%$ as well as better parent $402.78 \%$. The $\mathrm{F}_{1}$ hybrid JNA1/BVC-37 registered significant standard heterosis $(48.47 \%)$ for dry fruit weight plant ${ }^{-1}$. The highly significant gca effect revealed that LCA960, BVC37, LCA334, PantC1 and JNA1 were good general combiner among the male and female, respectively. The hybrid JNA1/BVC37 gave the highest yield for dry as well as green fruits along with highly significant sca effects.
\end{abstract}

Keywords: heterosis, heterobeltiosis, Capsicum annuum, chilli, combining ability

\section{Introduction}

India is the largest producer of chilli in the world owing to the availability of improved varieties. The role of hybrid seed in India is limited and the production of hybrid cultivars can be successful only if adequate quantities of hybrid seeds are produced at reasonably low cost. The hybrid seed production in chilli requires tedious process of emasculation and pollination. Chilli flowers are delicate in nature, resulting in flower drop or poor fruit set after emasculation. Moreover hand pollination increases the cost of hybrid seed production due to high labour cost. In the recent years hybrid cultivars have become popular and many farmers are producing hybrid seeds of hot pepper based on nuclear male sterility (Dash et al., 2001). Development of male sterility system is the only alternative to reduce the cost of seed production as it is being practiced in other vegetables. The male sterile (S-) cytoplasm has so far been most commonly utilized for commercial hybrid development in South Korea, China and India (Kim \& Kim, 2005; Kumar et al., 2007). However, rapid transfer of male sterile cytoplasm (nuclear diversification of CMS) in Indian genotypes has been a handicap due to the limited availability of maintainer allele $(r f)$ in chilli genotypes (Kumar et al., 2007) leading to restriction in the choice of the parents (Zhang, 2000). A major fertility restorer locus $(R f)$ is known to restore fertility of this S-cytoplasm, but it is influenced by temperature, quantitative trait loci (QTLs)/modifiers (Wang et al., 2004) and conditioned by an additional partial restoration (pr) locus (Lee et al., 2008a). This $p r$ locus is suspected to be either tightly linked to $R f$ locus or is third allele of $R f$ locus (Lee et al., 2008b). In Capsicum, very few stable nuclear-cytoplasmic male sterile (CMS) lines are known because male sterility expressions in CMS lines have been found to be temperature sensitive (Shifriss, 1997). Temperature alteration may induce a degree of variation in male sterility, ranging from complete to partial. Selfing as well as crossing of the male sterile individuals can be achieved through temperature manipulation. Therefore, breeders must use restorer as well as maintainer lines for various environments to secure a completely sterile female parent, but fully fertile hybrid.

One important aim of hybrid breeding is to exploit the heterosis effect appearing in the $F_{1}$. In development of high yielding varieties and hybrids of crop plants, the breeder often faces the problem of selecting parents and crosses. Common approach of selecting parents on the basis of per se always does not lead to fruitful results (Allard, 1960). The selection of parents has thus to be based on complete genetic information and knowledge of combining ability of potential parents. 
Combining ability analysis in this respect is considered to be an efficient technique not only for selection of desirable parents and crosses but also characterizes nature and magnitude of gene action in the expression of a trait. Knowledge about the type and amount of genetic effects is required for an efficient use of genetic variability of crops. The concept of good combining ability refers to the potential of a parental form for producing superior offspring by crossing with another parent for the breeding process. Information and exact study of combining ability can be useful in regard to selection of breeding methods and selection of lines for hybrid combination. Due to the numerous theoretical and practical advantages of this method, in recent years the choice of parental forms on the basis of combining ability has been extended.

Keeping in a view the present investigation was undertaken to identify the most heterotic male sterility based hybrids and generate information on general and specific combining ability effects for selection of parents (male sterile and restorer lines) for promising hybrid combinations.

\section{Materials and Methods}

\subsection{Plant Material}

The experimental material comprised of fifty one $\mathrm{F}_{1}$ hybrids developed from three females (CMS lines) viz., JNA1, ACA1 and ACA2 including seventeen male lines viz., SKS, PantC-1, G-4, K1-4C, 9608U, BVC-1, GUK-1, GUK-2, GUK-2-1, LCA334, LCA960, KDC-1-1, P. Jwala, KA-2, K1-4D, PSBR and BVC-37. Seventy one entries including twenty parents and their $51 \mathrm{~F}_{1}$ hybrids were evaluated in a Completely Randomized Block Design (CRBD) with two replications during kharif season 2009-2010 at JNKVV, Jabalpur (Madhya Pradesh). The plot size for each accession was $6 \mathrm{~m} \times 1.2 \mathrm{~m}$ where in both row-to-row and plant-to-plant spacing was $60 \mathrm{~cm} \times 60 \mathrm{~cm}$. The crop was raised as per the standard package of practice. The experiment was repeated thrice by transplanting the seedlings at the interval of twenty days. Timely management practices were followed to grow a good crop. Five plants were randomly selected/plot for recording data on days to first flower initiation, days to first fruit ripening, days to first harvest, number of pickings, number of fruits plant ${ }^{-1}$ number of primary branches and plant height $(\mathrm{cm})$, where as ten fruits were selected (two fruits from each randomly selected plant) for fresh weight fruit ${ }^{-1}(\mathrm{~g})$, dry weight fruit ${ }^{-1}(\mathrm{~g})$, fruit length $(\mathrm{cm})$, fruit diameter $(\mathrm{cm})$, pedicel length $(\mathrm{cm})$, petiole length $(\mathrm{cm})$, number of seeds fruit ${ }^{-1}$ and 1000 seed weight (g). For days to $50 \%$ flowering data was recorded on plot basis.

\subsection{Statistical Analysis}

The estimates of heterosis and combining ability were computed on mean performance of hybrids and their parents over two replications for each date of transplanting. Data on three transplanting dates were subjected to analysis for heterosis (Turner, 1953; Fonseca \& Patterson, 1968) and Line x Tester (Kempthorne, 1957) for combining ability analysis as per the standard procedure.

\subsubsection{Estimates of Heterosis}

Relative hybrid performance (in per cent) in comparison with the mean of both parents (mid-parent heterosis, $\mathrm{MPH})$, better parent, $\mathrm{BPH}$ and standard heterosis, $\mathrm{SH}$ were calculated as follows respectively:

$\mathrm{MPH}=(\mathrm{F} 1-\mathrm{MP}) / \mathrm{MP}) \times 100$, Where, $\mathrm{F} 1=$ mean performance of hybrid, $\mathrm{MP}=$ average performance of both parents.

$\mathrm{BPH}=(\mathrm{F} 1-\mathrm{BP}) / \mathrm{BP}) \times 100$, Where, $\mathrm{F} 1=$ mean performance of hybrid, $\mathrm{BP}=$ mean performance of better parents.

$\mathrm{SH}=(\mathrm{F} 1-\mathrm{SC}) / \mathrm{SC}) \times 100$, Where, $\mathrm{F} 1=$ mean performance of hybrid, $\mathrm{SC}=$ mean performance of standard check (Parvez, 2006).

\section{Results and Discussion}

\subsection{Analysis of Variance}

The estimates of mean square due to genotypes and parents were significant for all the traits (Table 1) indicating the presence of large amount of diversity among the genotypes and respective group of the material studied. Mean squares due to line, testers, and line $\mathrm{x}$ testers were also significant for most of the traits revealed the differences among females and males. The comparison of parents vs crosses was significant for all the traits suggesting presence of hybrid vigour for all the characters. 
Table 1. Analysis of variance for eighteen yield related traits in chilli

\begin{tabular}{|c|c|c|c|c|c|c|c|c|c|c|c|c|c|c|c|c|c|c|c|}
\hline Source & D.F. & DFI & DFF & DFR & DFH & $\mathrm{NOP}$ & NFP & FFW & DFW & $\mathrm{FL}$ & FD & PL & PTL & NPB & $\mathrm{PH}$ & NS & SW & FFWP & DFWP \\
\hline $\begin{array}{l}\text { Transplanting } \\
\text { dates }\end{array}$ & 02 & $311385^{* *}$ & $336396^{* *}$ & $8787 y^{* *}$ & $7735^{* *}$ & $0 \mathcal{B}^{* *}$ & $6997948^{* * *}$ & $599 *$ & $087^{7 * k}$ & $3499 \%$ & $045^{* *}$ & $1576^{* *}$ & $137 *$ & $1368^{* * *}$ & $9026^{* *}$ & $135817^{\text {** }}$ & $1676^{\text {tok }}$ & $1.7^{2 * *}$ & $0.11^{* *}$ \\
\hline Genotypes & 70 & $26400^{\circ *}$ & $2955^{* * *}$ & $4761^{* *}$ & $5421^{* *}$ & $0.12^{* *}$ & $4671025^{\text {** }}$ & $667^{\text {** }}$ & $030^{\text {kik }}$ & $23.49 \%$ & $024^{* *}$ & $158^{1 \% k}$ & $032 *$ & $231^{* *}$ & $23234 *$ & $119669^{2 *}$ & $3.16^{* *}$ & $06^{* * 4}$ & $0.04^{* * k}$ \\
\hline Parents & 19 & $2029 \%$ & $2035^{*}$ & $3902^{4 k}$ & $449^{\circ * k}$ & $026^{* *}$ & $18797.70^{\text {bok }}$ & $1445^{\text {** }}$ & $058^{\text {*i* }}$ & $4028 \% 8$ & $038^{* *}$ & $287^{* * *}$ & $0.14 *$ & $130^{1 k *}$ & $36931^{* *}$ & $94617^{* *}$ & $5.66^{* *}$ & $048^{* * *}$ & $0.3^{* *}$ \\
\hline Lines & 16 & $21,0^{2 *}$ & 2241 & $2499^{1 * k}$ & $3200^{7 * *}$ & $0.15^{* *}$ & $2104468^{* * *}$ & $14.12^{2 \%}$ & $0.2^{* *}$ & $41.04^{* * *}$ & $044^{* *}$ & $3.01^{\text {** }}$ & $0.11^{* *}$ & $129^{1 \% *}$ & $40251^{* *}$ & $85845^{* *}$ & $665^{* *}$ & $046+8$ & $0.3^{* *}$ \\
\hline Testers & 02 & 233 & 286 & $8186^{k *}$ & $4053^{* *}$ & 0.00 & $333733^{* *}$ & $050^{* k}$ & $007+8$ & $688 \%$ & $003^{* *}$ & $0.06^{*} 8$ & $006^{* *}$ & $1.00^{16 *}$ & $15811^{* k}$ & $58800^{* *}$ & $0.19^{* *}$ & $006 * 8$ & $0.00^{2 k}$ \\
\hline $\begin{array}{l}\text { Line vs } \\
\text { Testers }\end{array}$ & 01 & $450^{*}$ & 2243 & $177.75^{* *}$ & $251.06^{* *}$ & $256^{* *}$ & $1376686^{\text {‘k }}$ & $47.68^{\text {*k }}$ & $09^{\text {** }}$ & $9498^{\text {*3* }}$ & 000 & $637^{1 / k}$ & $084 *$ & $207^{* * *}$ & $260.60^{* *}$ & $306600^{1 / 2 k}$ & $0.72^{* *}$ & $1.79^{4 \%}$ & $0.11^{* *}$ \\
\hline $\begin{array}{l}\text { Parent } \\
\text { vsCrosses }\end{array}$ & 01 & $86427^{\text {t*k }}$ & $10142^{\text {k* }}$ & $955.43^{* *}$ & $67061 *$ & $066^{* * *}$ & $11642276^{2 * k}$ & $7486^{6 *}$ & $0.11^{* *}$ & $13^{7 * k}$ & $464^{* *}$ & $735^{* k}$ & $066^{* *}$ & $2241^{* *}$ & 406 & $505.2^{* *}$ & $673^{* *}$ & $7 . T^{* * *}$ & $049^{12 k}$ \\
\hline Crosses & 50 & 11.97 & 1336 & $3272^{20 k}$ & $4558^{20 k}$ & 005 & $349666^{12 *}$ & $235^{* *}$ & $020^{1 \% k}$ & $1755^{\text {** }}$ & $0.11^{* *}$ & $097^{\text {tik }}$ & $038 \%$ & $229^{* \% *}$ & $18486^{* *}$ & $1305.71^{* * k}$ & $213^{* *}$ & $054^{* *}$ & $0.0^{* 2 k}$ \\
\hline Enror & 140 & 1061 & 1080 & 0.89 & 057 & $00 B$ & 207.79 & 0.00 & 000 & 000 & 000 & 0.00 & 0.00 & 000 & 557 & 395 & 000 & 000 & 000 \\
\hline
\end{tabular}

$*, * *$ Significant at $\mathrm{p}=0.05$ and 0.01 , respectively.

$\mathrm{DFI}=$ Days to first flower initiation, $\mathrm{DFF}=$ Days to fifty per cent flowering, DFR $=$ Days to first fruit ripening, $\mathrm{DFH}=$ Days to first harvest, NOP $=$ Number of pickings, NFP $=$ Number of fruits plant ${ }^{-1}, \mathrm{FFW}=$ Fresh fruit weight fruit ${ }^{-1}(\mathrm{~g}), \quad \mathrm{DFW}=$ Dry fruit weight fruit $^{-1}(\mathrm{~g}), \mathrm{FL}=$ Fruit length $(\mathrm{cm}), \mathrm{FD}=$ Fruit diameter $(\mathrm{cm}), \mathrm{PL}=$ Pedicel length $(\mathrm{cm})$, PTL $=$ Petiole length $(\mathrm{cm}), \mathrm{NPB}=$ Number of primary branches, $\mathrm{PH}=$ Plant height $(\mathrm{cm})$, $\mathrm{NS}=$ Number of seeds fruit ${ }^{-1}, \mathrm{TSW}=1000$ seed weight $(\mathrm{g}), \mathrm{FFWP}=$ Fresh fruit weight plant $^{-1}(\mathrm{~kg}), \mathrm{DFWP}=$ Dry fruit weight plant $^{-1}(\mathrm{~kg})$.

\subsection{Mean Performance of Parents and Hybrids}

The range for mean performance and various heterotic effects as well as promising crosses identified on the basis of these two parameters are narrated in Table 2. The maximum range of mean performance was observed for number of fruits plant ${ }^{-1}$ for both, parents $(71.67$ - 388.33) and hybrids $(162.33-565.17)$ along with high heterotic effects over mid parent $(21.75$ - 658.29), better parent $(-1.8-651.37)$ and standard check $(43.02-397.94)$. Genotypes BVC-37, BVC-1, GUK2-1, LCA960 and K1-4D registered as better parents based on per se performance for dry fruit weight plant ${ }^{-1}$. KA-2 exhibited better parent for days to first flower initiation and days to fifty per cent flowering, JNA1 for days to first fruit ripening and days to first harvest, PSBR, K1-4D, KA-2, KDSC1-1 and LCA960 for number of pickings, PantC1 for number of fruits plant ${ }^{-1}$, LCA960 for fresh weight fruit $^{-1}$, dry weight fruit ${ }^{-1}$, number of seeds fruit ${ }^{-1}$ and 1000 seed weight, SKS for fruit length, GUK2-1 for fruit diameter, G-1 for pedicel length, BVC-37 for petiole length, fresh fruit weight plant ${ }^{-1}$ and dry fruit weight plant ${ }^{-1}$, $9608 \mathrm{U}$ for number of primary branches plant ${ }^{-1}$.

Table 2. Five bests parents for eighteen characters in chilli

\begin{tabular}{|c|c|c|c|c|c|c|}
\hline \multirow{3}{*}{ Characters } & \multicolumn{5}{|c|}{ Range } & \multirow[t]{3}{*}{ Betterparents (Basedon perse performance) } \\
\hline & \multicolumn{2}{|c|}{ Perseperformance } & \multicolumn{3}{|c|}{ Heterosis } & \\
\hline & Parents & Crosses & MP & $\mathrm{BP}$ & $\mathrm{SH}$ & \\
\hline Days to first flower initiation & $33.17-44.33$ & $32.00-41.83$ & -18.22 to 5.24 & -24.06 to 0.40 & 1.59 to 32.80 & $\mathrm{KA}-2(33.17), \mathrm{K} 1-4 \mathrm{C}(35.50), \operatorname{ACA} 1$ (36.17),ACA2 (37.50), $\operatorname{PantC} 1$ (37.83) \\
\hline Days to fifty per cent flowering & $37.00-48.33$ & $35.33-45.67$ & -18.08 to 4.18 & -23.45 to 0.74 & -0.93 to 28.04 & KA-2 (37.00),K1-4C (39.17), GUK2-1 (40.33),ACA1 (40.50), $\operatorname{PantCl}(41.00)$ \\
\hline Days to first fruit ripening & 93.33-105.5 & $82.67-94.33$ & -13.69 to 0.74 & -21.33 to 0.89 & 0.81 to 15.04 & JNA1 (85.83),ACA1 (93.00), PSBR (93.33),K1-4D(93.50),KDC-1 (94.33) \\
\hline Days to first harvest & $114.17-128.67$ & $105.0-117.67$ & -11.53 to 0.65 & -18.39 to 1.02 & -0.49 to 15.93 & JNA1 (108.33),ACA1(113.33),K1-4D (114.17),PSBR(114.33),GUK-2(115.50) \\
\hline Number of pickings & 2.33-3.00 & 2.33-3.17 & 0.00 to 35.71 & -5.88 to 35.71 & -22.22 to 5.56 & $\operatorname{PSBR}(3.00), \mathrm{K} 1-4 \mathrm{D}(3.00), \mathrm{KA}-2(3.00), \mathrm{KDSCl}-1(3.00), \operatorname{LCA} 960(3.00)$ \\
\hline Number of fruits plant ${ }^{-1}$ & $71.67-388.33$ & $162.33-565.17$ & 21.75 to 658.29 & -1.8 to 651.37 & 43.02 to 397.94 & PaniCl (388-33),PSBR (286.33), GUK-2-1 (243.50), BVC-37 (193),9608U(144.67) \\
\hline Fresh fruit weight fruit ${ }^{-1}(\mathrm{~g})$ & $1.59-8.20$ & $1.81-4.91$ & -54.47 to 85.26 & -69.17 to 69.05 & -83.33 to -54.12 & LCA960(8.20),BVC-1 (8.00),K1-4D(7.97),BVC-37(7.53),KDCl-1 (6.13) \\
\hline Dry fruit weight fruit ${ }^{-1}(\mathrm{~g})$ & $0.48-1.97$ & $0.41-1.50$ & -1.09 to 84.38 & -62.51 to 70.94 & -84.81 to -44.15 & LCA960(1.97),BVC-37(1.92),BVC-1 (1.63),SKS(1.10), GUK-2-1 (0.98) \\
\hline Fruit length (cm) & $6.10-20.43$ & $4.85-17.52$ & -25.88 to 86.13 & -51.25 to 66.37 & -67.39 to 17.72 & SKS(20.43),BVC-37(13.80),K1-4D(12.36),BVC-1 (12.26), GUK-1 (12.06) \\
\hline Fruit diameter $(\mathrm{cm})$ & $0.93-2.24$ & $0.77-1.96$ & -50.69 to 19.59 & -54.05 to- -1.42 & -49.51 to 28.74 & GUK2-1 (2.24),BVC-37(1.99),LCA960(1.99),K1-4C(1.64),KDC-1-1 (1.59) \\
\hline Pedicel length (cm) & $2.85-6.96$ & $2.53-4.91$ & -38.99 to 52.80 & -54.69 to 46.72 & -48.68 to -0.30 & G4(6.90), GUK-2 (5.20),SKS(5.00),KDC-1-1 (4.65),BVC-37 (4.54) \\
\hline Petiole length (cm) & $1.36-2.06$ & $1.10-2.63$ & -32.74 to 59.98 & -37.47 to 31.77 & -29.27 to 68.80 & BVC-37(2.06), GUK-1 (2.05),PJwala(1.95),KA-2(1.93), GUK-2 (1.41) \\
\hline Number of primary branches & $3.79-5.79$ & $3.79-7.89$ & -20.87 to 83.88 & -34.53 to 79.12 & -5.01 to 97.70 & 9608U(5.79), $\mathrm{PantCl}(5.79), \mathrm{BVC}-37(529), \mathrm{K} 1-4 \mathrm{D}(4.79), \mathrm{KA}-2$ (4.79). \\
\hline Plant height (cm) & $41.83-83.83$ & $41.83-81.17$ & -30.85 to 48.31 & -37.41 to 44.48 & -31.98 to 31.98 & SKS(83.83),BVC-37(81.83),PSBR(76.17),K1-4D(70.50),LCA960(68.50) \\
\hline Number of seeds fruit ${ }^{-1}$ & $57.50-120.33$ & $32.83-124.50$ & -54.41 to 52.92 & -68.98 to 40.68 & -91.11 to- -20.78 & LCA960(120.33),K1-4C(105.83),BVC-1 (101.83),K1-4D(95.00),PSBR (92.83) \\
\hline 1000 seed weight $(\mathrm{g})$ & 3.249 .44 & $3.36-7.68$ & -36.05 to 69.38 & -45.65 to 60.88 & -38.90 to 39.62 & LCA960(9.44),BVC-1 (8.25), GUK-2 (5.53),BVC-37 (5.46), GUK-1 (5.38) \\
\hline Fresh fruit weight plant ${ }^{-1}(\mathrm{~kg})$ & 0.241 .81 & $0.57-2.27$ & -1.15 to 495.88 & -42.79 to 409.86 & -63.11 to 47.88 & BVC-37(1.81),BVC-1 (1.39),GUK2-1(1.17),LCA960(1.12),K1-4D(0.90) \\
\hline Dry fruit weight plant ${ }^{-1}(\mathrm{~kg})$ & $0.06-0.45$ & 0.140 .57 & -0.92 to 493.44 & -42.79 to 402.78 & -62.88 to 48.47 & BVC-37(0.45),BVC-1 (0.35), GUK2-1 (0.30),LCA960 (0.28),K1-4D(0.22) \\
\hline
\end{tabular}




\subsection{Heterotic Effects of Crosses}

Most heterotic crosses are presented in Table 3 revealed that none of the hybrids showed desirable standard heterosis for days to first flower initiation, days to first fruit ripening, fresh weight fruit ${ }^{-1}$, dry weight fruit ${ }^{-1}$, pedicel length and number of seeds fruit ${ }^{-1}$. However, one hybrid each for days to fifty per cent flowering and days to first harvest registered desirable standard heterosis. While 36 crosses for number of pickings, all 51 for number of fruits plant ${ }^{-1}$ and number of primary branches, 2 for fruit length, 1 for fruit diameter, 40 for petiole length, 23 for plant height, 26 for 1000 seed weight and 12 each for fresh fruit weight plant ${ }^{-1}$ and dry fruit weight plant ${ }^{-1}$ exhibited desirable standard heterosis. For fruit length and fruit diameter, most of the crosses were not consistent for their various heterotic effects. These findings are concordance with Joshi et al. (1995), Patel et al. (1997) and Patel et al. (2001).

Table 3. Most heterotic crosses for eighteen characters in chilli for dry fruit yield

\begin{tabular}{|c|c|c|c|c|c|}
\hline \multirow[t]{2}{*}{ Characters } & \multicolumn{2}{|c|}{$\begin{array}{c}\text { No. of hybrids having } \\
\text { significant heterotic } \\
\text { effects over SH }\end{array}$} & \multicolumn{3}{|c|}{ Best hybrids based on } \\
\hline & $+\mathrm{Ve}$ & $-\mathrm{Ve}$ & MP & $\mathrm{BP}$ & $\mathrm{SH}$ \\
\hline Days to first flower initiation & 51 & 0 & JNA1/G-4 & ACA1/BVC-37 & ACA1/GUK2-1 \\
\hline Days to fifty per cent flowering & 50 & 1 & ACA2/BVC-37 & ACA2/BVC-37 & ACA1/GUK2-1 \\
\hline Days to first fruit ripening & 51 & 0 & ACA2/LCA334 & JNA1/SKS & JNA1/PantC1 \\
\hline Days to first harvest & 50 & 1 & JNA1/PantC1 & JNA1/SKS & JNA1/PantC1 \\
\hline Number of pickings & 36 & 15 & JNB1/BVC-37 & JNB1/BVC-37 & JNB1/BVC-37 \\
\hline Number of fruits plant ${ }^{-1}$ & 51 & 0 & ACA1/LCA334 & ACA1/LCA334 & ACA12/PantC1 \\
\hline Fresh fruit weight fruit ${ }^{-1}(\mathrm{~g})$ & 0 & 51 & JNA1/KA2 & JNA1/PantC1 & JNA1/LCA960 \\
\hline Dry fruit weight fruit ${ }^{-1}(g)$ & 0 & 51 & JNA1/GUK2-1 & JNA1/PantC1 & JNA1/GUK2-1 \\
\hline Fruit length $(\mathrm{cm})$ & 2 & 49 & JNA1/GUK2-1 & JNA1/GUK2-1 & JNA1/SKS \\
\hline Fruit diameter (cm) & 1 & 50 & JNA1/LCA960 & JNA1/LCA960 & JNA1/LCA960 \\
\hline Pedicel length (cm) & 0 & 51 & JNA1/LCA334 & JNA1/LCA334 & JNA1/GUK2-1 \\
\hline Petiole length (cm) & 40 & 11 & ACA2/GUK2-1 & $\mathrm{ACA} 2 / \mathrm{K} 1-4 \mathrm{C}$ & ACA2/SKS \\
\hline Number of primary branches & 51 & 0 & ACA1/LCA334 & $\mathrm{ACA} 2 / \mathrm{K} 1-4 \mathrm{C}$ & ACA1/LCA334 \\
\hline Plant height (cm) & 23 & 28 & ACA1/LCA334 & ACA1/LCA334 & JNA1/SKS \\
\hline Number of seeds fruit ${ }^{-1}$ & 0 & 51 & JNA1/BVC-37 & JNA1/BVC-37 & JNA1/BVC-37 \\
\hline 1000 seed weight (g) & 26 & 25 & ACA1/9608U & ACA1/9608U & ACA1/GUK2 \\
\hline Fresh fruit weight plant ${ }^{-1}(\mathrm{~kg})$ & 12 & 39 & ACA1/LCA334 & ACA1/LCA334 & JNA1/BVC-37 \\
\hline Dry fruit weight plant $^{-1}(\mathrm{~kg})$ & 12 & 39 & ACA1/LCA334 & ACA1/LCA334 & JNA1/BVC-37 \\
\hline
\end{tabular}

A perusal of the top heterotic crosses revealed that none of the cross was consistent for all the traits. Among the 51 $\mathrm{F}_{1}$ hybrids studied 8 crosses viz., JNA1/BVC-37 (48.47\%), JNA1/PantC1 (25.76\%), JNA1/LCA334 (25.33\%), JNA1/LCA960 (24.02\%), JNA1/PSBR (22.77\%), JNA1/GUK-2-1(16.59\%), ACA2/LCA960 (13.10\%), ACA1/LCA960 (6.99\%) showed significant positive heterosis for dry fruit weight plant ${ }^{-1}$ over standard check VNR 332 (Table 4). However, other thirty nine crosses registered negative standard heterosis and remnant four crosses recorded non significant positive standard heterosis.

Table 4. Heterosis for dry and green fruit yield plant ${ }^{-1}$ for 8 best hybrids in chilli

\begin{tabular}{|c|c|c|c|c|c|c|c|c|}
\hline \multirow{3}{*}{ F1 Crosses } & \multicolumn{8}{|c|}{ Heterosis over parents/check } \\
\hline & \multicolumn{4}{|c|}{ Dry fruit yield } & \multicolumn{4}{|c|}{ Green fruit yield } \\
\hline & Mean & Mid parent & Better parent & VNR332 (check) & Mean & $\begin{array}{c}\text { Mid } \\
\text { parent }\end{array}$ & $\begin{array}{l}\text { Better } \\
\text { parent }\end{array}$ & $\begin{array}{c}\text { VNR332 } \\
\text { (check) }\end{array}$ \\
\hline JNA1/BVC-37 & 0.57 & $104.82 * *$ & $25.46 * *$ & $48.47 * *$ & 2.27 & $103.44 * *$ & $25.37 * *$ & $47.88 * *$ \\
\hline JNA1/PantC-1 & 0.48 & $229.14 * *$ & $152.63 * *$ & $25.76 * *$ & 1.92 & $224.68 * *$ & $151.86^{* *}$ & $25.24 * *$ \\
\hline JNA1/LCA334 & 0.48 & $491.75 * *$ & $370.49 * *$ & $25.33 * *$ & 1.91 & $482.74 * *$ & $355.56^{* *}$ & $24.92 * *$ \\
\hline JNA1/LCA960 & 0.47 & $148.03 * *$ & $69.05 * *$ & $24.02 * *$ & 1.90 & $147.02 * *$ & $69.90 * *$ & $24.05 * *$ \\
\hline JNA1/PSBR & 0.47 & $252.20 * *$ & $185.71 * *$ & $22.27 * *$ & 1.88 & $249.92 * *$ & $187.72 * *$ & $22.42 * *$ \\
\hline JNA1/GUK-2-1 & 0.44 & $124.37 * *$ & $50.85^{* *}$ & $16.59 * *$ & 1.78 & $123.43 * *$ & $51.70 * *$ & $16.21 * *$ \\
\hline ACA2/LCA960 & 0.43 & $133.33 * *$ & $54.17 * *$ & $13.10 * *$ & 1.73 & $133.33 * *$ & $54.40 * *$ & $12.73 * *$ \\
\hline ACA1/LCA960 & 0.41 & 153.89 & $45.83 * *$ & $06.99 *$ & 1.63 & $153.37 * *$ & $45.75 * *$ & $06.42 *$ \\
\hline
\end{tabular}




\subsection{GCA and SCA Effects}

The gca effect (Table 5) revealed that LCA960, BVC-37, LCA334, PantC1 and JNA1 were good general combiner among the male and female, respectively. Highly significant gca effect recorded by SKS, G-4, PSBR and ACA1 for days to first flower initiation, SKS, G-4, PSBR and ACA1 for days to fifty per cent flowering, JNA1 for days to first harvest, LCA334, LCA960, PSBR, BVC-37 and JNA1 for number of pickings, SKS, BVC-37, GUK-1, GUK-2, LCA960, KDC-1-1, KA-2, K1-4D, BVC-37 and JNA1 for fresh weight fruit ${ }^{-1}$, SKS, 9608U, BVC-1, GUK-1, GUK-2-1, KDC-1-1, BVC-37 and JNA1 for dry weight fruit ${ }^{-1}$, SKS, K1-4C, BVC-1, KDC-1-1, K1-4D, BVC-37 and JNA1 for fruit length, 9608U, BVC-1, GUK-2-1, LCA960, K1-4D, BVC-37 and JNA1 for fruit diameter, PantC-1, K1-4C, 9608U, GUK-2-1, LCA334, P.JWALA, PSBR, BVC-37 and JNA1 for pedicel length, SKS, G4, K1-4C, GUK-4, GUK-2, GUK-2-1, KDC1-1, K1-4D, BVC-37, and ACA1 for petiole length, SKS, K1-4C, GUK-1, GUK-2, LCA334, KDC1-1, BVC-37 and JNA1 for number of primary branches, BVC-37 and JNA1 for number of seeds fruit ${ }^{-1}$, SKS, PantC-1, G-4, 9608U, BVC-1, GUK-1, GUK-2, K1-4D, BVC-37, ACA1, and ACA2 for 1000 seed weight.

Table 5. Estimates of gca effects of parents for different traits in chilli

\begin{tabular}{|c|c|c|c|c|c|c|c|c|c|c|c|c|c|c|c|c|c|c|c|}
\hline \multirow{2}{*}{$\begin{array}{l}\text { Parents } \\
\text { Tester }\end{array}$} & \multicolumn{19}{|c|}{ Characters } \\
\hline & DFI & DFF & DFR & $\mathrm{DFH}$ & NOP & NFP & FFW & DFW & FL & FD & PL & PIL & $\mathrm{NPB}$ & $\mathrm{PH}$ & NS & SW & FFWP & DFWP & NPY \\
\hline SKS & $646^{* 2 k}$ & $646^{* * *}$ & $-1.45^{* \%}$ & $-193^{* *}$ & $-0.04^{* * k}$ & $-631^{\text {** }}$ & $0.16^{* *}$ & $0.07^{+* *}$ & $38^{\circ \% *}$ & $-0.05^{* * k}$ & $-0.14^{* * k}$ & $0.12^{* *}$ & $0.07^{* * *}$ & 3873 & 056 & $047^{* * *}$ & $0.05^{* * *}$ & $0.013^{\text {*ak }}$ & $0.11^{\text {** }}$ \\
\hline PantC-1 & 0.46 & 0.461 & $-1.45^{* \%}$ & $-293^{* * *}$ & $-0.039^{1 k k}$ & 17535 & $-0.48^{* k}$ & $-0.02^{\text {*ak }}$ & $-132^{2 * *}$ & $-0.08^{* * k}$ & $0.16^{\text {*k }}$ & $-038^{* * k}$ & $-0.46^{* * k}$ & $-0.46^{* k}$ & $-844^{* *}$ & $0.11^{\text {*k }}$ & $031^{\text {*** }}$ & $0.08^{1 * k}$ & $0.70^{\mathrm{kek}}$ \\
\hline G-4 & $229^{*}$ & $229^{*}$ & 1.88 & 207 & $-0.04^{* * *}$ & $-7331^{* *}$ & $-052^{* \%}$ & $-0.08^{* k *}$ & $-1,89 \%$ & $-0.06^{* * *}$ & $-034^{* *}$ & $0.11^{* *}$ & $-0.60^{\circ \%}$ & $-1096^{\text {*ak }}$ & 1.89 & $0.42^{2 * *}$ & $-0.4 * \%$ & $-0.11^{\text {** }}$ & $-0.99 \%$ \\
\hline $\mathrm{K} 1-4 \mathrm{C}$ & $-037^{* *}$ & $-037^{* * *}$ & 138 & 1.069 & $-0.040^{1 \mathrm{kek}}$ & $-11215^{\text {*k }}$ & $-020 \%$ & $-0.11^{* *}$ & $1.84^{* * *}$ & $-0.03^{* * k}$ & $0.09^{9 * k}$ & $0.01 *$ & $1.17^{7 \% *}$ & $-5.13^{* * k}$ & $-928^{* *}$ & $-0.07^{* * *}$ & $-0.48^{* *}$ & $-0.12^{\% * k}$ & $-1.07^{\text {tw }}$ \\
\hline $9608 \mathrm{U}$ & $-0.87^{* *}$ & $-0.87^{* * *}$ & 122 & 157 & $-0.039^{1 k *}$ & $-10098^{* 2 k}$ & $-032^{\% *}$ & $026^{* * *}$ & $-1.6^{* * *}$ & $0.14^{* * *}$ & $0.04^{* * k}$ & $-0.03^{* * *}$ & $-0.43^{* * *}$ & $-0.46^{* \% k}$ & 9.89 & $0.6 \% *$ & $-045^{* *}$ & $-0.11^{\text {** }}$ & $-1.00^{\text {set }}$ \\
\hline BVC-1 & $-121^{* *}$ & $-12^{* * *}$ & $-028^{* * *}$ & $-1.10^{\text {wk }}$ & $-0.039^{* k}$ & $-7681^{* *}$ & $0.19^{1 \% *}$ & $0.16^{\text {*** }}$ & $090^{\mathrm{pok}}$ & $0.02^{2 \% *}$ & $-0.16^{* k}$ & $-025^{* *}$ & $-050^{0 \%}$ & $-3.3^{* k}$ & $-1294^{* * *}$ & $0.05^{* * *}$ & $-021^{* *}$ & $-0.05^{* *}$ & $-0.47^{* *}$ \\
\hline GUK-1 & $-1.71^{\text {\%* }}$ & $-1.72^{* * *}$ & 0.72 & 090 & $-0.039^{1 * k}$ & $-70.15^{* *}$ & $0.11^{* * *}$ & $0.007^{* * *}$ & 0.003 & $-0.02^{* * *}$ & $-0.09 \%$ & $029^{\text {kk }}$ & $0.14^{* * *}$ & $4.46^{* * k}$ & 039 & $0.16^{* * *}$ & $-0.19^{* *}$ & $-0.047^{\text {t*k }}$ & $-0.42^{\text {** }}$ \\
\hline GUK-2 & $-204^{* * *}$ & $-200^{* *}$ & 0.72 & 157 & $-0.039^{1 *}$ & $-8748^{\text {*** }}$ & $0.16^{* *}$ & $-021^{* *}$ & $-1.01^{* *}$ & $-0.11^{* *}$ & $-0.03^{* *}$ & $031^{\text {** }}$ & $1.04 * *$ & 137 & $-0.44^{* *}$ & $027^{7 * *}$ & $-026^{* *}$ & $-0.07^{* * *}$ & $-057^{\text {** }}$ \\
\hline GUK-2-1 & $-204^{* *}$ & $-203^{* * k}$ & $-1.77^{* *}$ & $-0.7^{* * *}$ & $-0.039^{k * k}$ & 111.19 & $-0.18^{\text {*k }}$ & $0.17^{* * *}$ & $-054 *$ & $0.07^{* * *}$ & $0.47^{\text {*k }}$ & $0.15^{* *}$ & $-0.06^{* * *}$ & $-096^{* * k}$ & 856 & $-0.67^{* \%}$ & $035^{* * k}$ & $0.09^{2 k}$ & $0.78^{\text {*ak }}$ \\
\hline LCA334 & $-0.04^{* *}$ & $-0.039 *$ & $-1.77^{* *}$ & $-1.60^{p k}$ & $0.127^{\text {*k }}$ & 17635 & $-0.4 * \%$ & $-0.16^{\text {** }}$ & $-1.03^{* *}$ & $-0.17^{* * *}$ & $0.43^{* *}$ & $-0.04^{* * *}$ & $05^{7 * *}$ & 837 & $-8.11^{* *}$ & $-059 \%$ & $0.40^{\circ \%}$ & $0.10^{2 k}$ & $0.88^{* k *}$ \\
\hline LCA960 & 0.627 & 0.627 & $-1.77^{* * *}$ & $-127^{* * *}$ & $0.127^{\text {*k }}$ & 9235 & $054^{* *}$ & $-0.002^{* * *}$ & $-12^{* *}$ & $036^{* *}$ & $-05^{\text {tok }}$ & $-030^{\circ \% k}$ & $-0.40^{\circ \%}$ & 0.04 & 123 & $-027^{* * k}$ & $0.60^{\circ \%}$ & $0.15^{* *}$ & $133^{* *}$ \\
\hline KDC-1-1 & $-154^{* *}$ & $-153^{3 * *}$ & 1.05 & 057 & $-0.039^{1 * k}$ & $-5998^{* \% *}$ & $032^{2 * *}$ & $0.02^{2 * *}$ & $098^{* * *}$ & $-0.10^{* \%}$ & $-0.07^{* * k}$ & $0.02^{* *}$ & $0.17^{7 * *}$ & 537 & 4.73 & $-058^{\text {*ik }}$ & $-0.10^{2 \%}$ & $-0.02^{* * *}$ & $-023^{\text {*क }}$ \\
\hline P. Jwala & $-137^{* * *}$ & $-137^{* * \%}$ & 122 & 240 & $-0.039^{k * k}$ & $4548^{* * *}$ & $-0.76^{* * k}$ & $-022^{* *}$ & $-1.80^{20 \%}$ & $-0.07^{* * *}$ & $0.11^{* * *}$ & $-025^{* *}$ & $-02^{* * *}$ & $-5.46^{* \% k}$ & $-15.94^{* * *}$ & $-035^{* *}$ & $-0.42^{* *}$ & $-0.10^{k \%}$ & $-0.93^{\text {** }}$ \\
\hline KA-2 & $-137^{* *}$ & $-137^{* * *}$ & $-0.77^{* * *}$ & $-243^{* * *}$ & $-0.039^{1 * k}$ & $-6465^{\% *}$ & $1.11^{\text {** }}$ & $-0.045^{* * *}$ & $-0.72^{2 *}$ & $-0.10^{1 \% k}$ & $-028^{* k *}$ & $-0.03^{* * *}$ & $-026^{* * *}$ & $-3.79 \%$ & $-7.61^{* *}$ & $-032^{\% *}$ & $0.17^{* * *}$ & $0.04^{* * k}$ & $038^{10 *}$ \\
\hline $\mathrm{K} 1-4 \mathrm{D}$ & $-0.71^{* *}$ & $-0.72^{2 * *}$ & 022 & 0.74 & $-0.039^{* *}$ & $-5598^{* * *}$ & $0.85^{* *}$ & $-0.088^{* * k}$ & $253^{* * *}$ & $0.14^{* * *}$ & $-0.07^{* * k}$ & $025^{* *}$ & $-033^{* * *}$ & 7.04 & 16.06 & $02^{2 * *}$ & $0.11^{\text {** }}$ & $0.03^{* *}$ & $025^{\text {** }}$ \\
\hline PSBR & $27^{10 k}$ & $279^{* * *}$ & $-0.45^{* *}$ & $-0.77^{* * *}$ & $0.127^{* k *}$ & 120.02 & $-0.81^{* *}$ & $-0.010^{20 *}$ & $-0.13^{* * *}$ & $-0.15^{* k}$ & $0.03^{* * *}$ & $-0.09 \%$ & $-0.13^{* * *}$ & 721 & $-3.11^{*}$ & $-025^{* *}$ & $0.08 \%$ & $0.02^{2 * *}$ & $0.19^{k k}$ \\
\hline BVC-37 & 0.62 & 0.63 & 138 & 190 & $0.127^{* * k}$ & 7802 & $024 * *$ & $0252^{\text {** }}$ & $1.15^{* * *}$ & $021^{* * *}$ & $09^{9 * k}$ & $0.12^{* * *}$ & $024^{* * *}$ & 204 & $2256^{*}$ & $0.76^{* * *}$ & $048^{* * *}$ & $0.12^{2 * k}$ & $1.07^{\text {tek }}$ \\
\hline SED & 0.60 & 0.60 & 30.75 & 0.84 & 0.10 & 14.82 & 0.06 & 0.003 & 0.06 & 0.01 & 0.05 & 0.08 & 0.00 & 251 & 210 & 0.00 & 0.04 & 0.01 & 0.10 \\
\hline CD 5\% & 1.19 & 1.19 & 61.78 & 1.68 & 021 & 29.77 & 0.119 & 0.007 & 0.116 & 0.01 & 0.10 & 0.08 & 0.00 & 5.05 & 422 & 0.00 & 29.77 & 0.02 & 020 \\
\hline $\mathrm{CD} 1 \%$ & 159 & 159 & 8236 & 224 & 028 & 39.69 & 0.15 & 0.009 & 0.16 & 0.02 & 0.13 & 0.10 & 0.00 & 673 & 5.63 & 0.00 & 39.69 & 0.03 & 026 \\
\hline \multicolumn{20}{|l|}{ Lines } \\
\hline JNA1 & 0.04 & 0.039 & 0.441 & $0.8^{*}$ & $0.078^{* * *}$ & $-15.78^{\text {*** }}$ & $0.81^{* *}$ & $0.19^{1 * *}$ & $1.83^{* *}$ & $0.02^{2 * *}$ & $050^{\mathrm{pek}}$ & $-0.12^{* *}$ & $0.18^{* * *}$ & 433 & $1845^{\text {** }}$ & $-0.16^{* *}$ & $029^{9 \%}$ & $0.07^{* k *}$ & $0.64^{* * k}$ \\
\hline ACA1 & $033^{*}$ & $033^{*}$ & 0.147 & $-027^{* * *}$ & $-0.039^{* *}$ & 22598 & $-0.60^{\mathrm{sk}}$ & $-0.13^{* * *}$ & $-139^{* * *}$ & $-0.02^{* * k}$ & $-0.14^{* *}$ & $0.017^{\text {jok }}$ & $0.03 * *$ & $-19^{* * *}$ & $-9.17^{7 * *}$ & $0.08^{8 * \%}$ & $-0.18^{* *}$ & $-0.05^{* *}$ & $-0.39 \%$ \\
\hline ACA2 & $-037^{* *}$ & $-037^{* * *}$ & $-0.59 \%$ & $-059^{\circ * k}$ & $-0.034^{* *}$ & $-6.814^{* *}$ & $-020 \%$ & $-0.057^{\text {*k }}$ & $-0.45^{* *}$ & $0.005^{* *}$ & $-036^{* * k}$ & $0.106^{\text {** }}$ & $-021^{* *}$ & $-241^{* *}$ & $-928^{* *}$ & $0.08^{* * *}$ & $-0.11^{* *}$ & $-0.03^{* *}$ & $-024^{*}$ \\
\hline SED & 025 & 0250 & 1292 & 0351 & 0.043 & 622 & 0.023 & 0.002 & 0.024 & 0.003 & 0.020 & 0.015 & 0.000 & 1.06 & 0.82 & 0.000 & 0,02 & 0.01 & 0.04 \\
\hline CD 5\% & 050 & 0502 & 25.95 & 0.705 & 0.087 & 1550 & 0.048 & 0.003 & 0.048 & 0.006 & 0.041 & 0.031 & 0.000 & 212 & 1.78 & 0.000 & 0.04 & 0.01 & 0.08 \\
\hline $\mathrm{CD} 1 \%$ & 0.67 & 0.669 & 34,60 & 094 & 0.116 & 1667 & 0.64 & 0.004 & 0.065 & 0.008 & 0.054 & 0.042 & 0.000 & 283 & 236 & 0.000 & 0.05 & 0.01 & 0.11 \\
\hline
\end{tabular}

$\mathrm{DFI}=$ Days to first flower initiation, $\mathrm{DFF}=$ Days to fifty per cent flowering, DFR $=$ Days to first fruit ripening, $\mathrm{DFH}=$ Days to first harvest, NOP $=$ Number of pickings, NFP $=$ Number of fruits plant ${ }^{-1}, \mathrm{FFW}=$ Fresh fruit weight fruit ${ }^{-1}(\mathrm{~g}), \quad \mathrm{DFW}=$ Dry fruit weight fruit $^{-1}(\mathrm{~g}), \mathrm{FL}=$ Fruit length $(\mathrm{cm}), \quad \mathrm{FD}=$ Fruit diameter $(\mathrm{cm})$, PL $=$ Pedicel length $(\mathrm{cm}), \mathrm{PTL}=$ Petiole length $(\mathrm{cm}), \mathrm{NPB}=$ Number of primary branches, $\mathrm{PH}=$ Plant height $(\mathrm{cm})$,

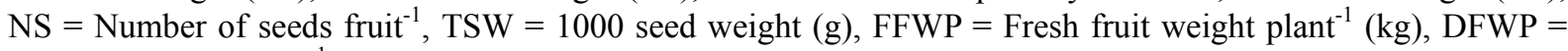
Dry fruit weight plant ${ }^{-1}(\mathrm{~kg}), \mathrm{NPY}=$ Net plot yield $(\mathrm{kg})$.

Among the lines and testers genotypes SKS, PantC-1, GUK2-1, LCA334, LCA960, KA-2, K1-4D, PSBR, BVC-37 and JNA1 were found to be best general combiner for traits fresh fruit weight plant ${ }^{-1}$, dry fruit weight 
plant $^{-1}$ and net plot yield. Therefore, these genotypes can be utilized for producing promising hybrids in hybridization programme.

In general, genotype SKS, BVC-37, LCA334 and JNA1 male and female respectively were found to be good general combiner for most of the characters. The traits like primary branches have direct bearing on number of fruits and numbers of fruits have positive impact on yield, hence these four genotypes viz., SKS, BVC-37, LCA334 (male) and JNA1 (female) can be used to produce the best hybrid. JNA1/BVC-37, JNA1/PSBR, JNA1/SKS, JNA1/9608U and JNA1/GUK-2 were the best five crosses each in respect to per se performance and significant sca effects (Table 6). Among the five best crosses, highly significant sca effects were recorded by crosses JNA1/BVC-37 and JNA1/PSBR for fresh fruit weight plant ${ }^{-1}$ and dry fruit weight plant ${ }^{-1}$. It was evident that the cross with high $\mathrm{x}$ low gca effect could produce desirable transgressive segregants. JNA1/BVC-37, JNA1/PSBR, JNA1/SKS, JNA1/9608U and JNA1/GUK-2 showed highly significant sca effects with parents having high $\mathrm{x}$ low general combiners, it might be attributed to sizeable portion of additive $\mathrm{x}$ additive gene action.

Table 6. Estimates of sca effects of crosses for different traits in chilli

\begin{tabular}{|c|c|c|c|c|c|c|c|c|c|c|c|c|c|c|c|c|c|c|c|}
\hline Crosses & DFI & DFF & DFR & DFH & NOP & NFP & FFW & DFW & FL & FD & PL & PTL & NPB & $\mathrm{PH}$ & NS & SW & FFWP & DFWP & NPY \\
\hline JNA1/SKS & 022 & $-245^{* *}$ & $-7.84^{* * *}$ & $-680^{\circ / 2 k}$ & $-0.6^{3 *}$ & 3.68 & $053^{* *}$ & $030^{\text {pok }}$ & $250^{\circ k k}$ & $0.04^{* * *}$ & 433 & $-0.18^{* * *}$ & $-0.75^{\text {*k }}$ & $1286^{* * *}$ & $20.000^{\text {ek }}$ & $0.6^{2 * k}$ & 0.09 & 0.02 & 261 \\
\hline JNA1/PantC-1 & $-395^{* *}$ & -1.45 & $-851^{\text {*k }}$ & $-103^{* * k}$ & $0.04^{* * *}$ & $-66.16^{\text {*k }}$ & $0.2^{2 * *}$ & $0.13^{* *}$ & $121^{* * *}$ & 0.01 & 333 & -0.05 & $0.79^{k * k}$ & 020 & 45 & $-028^{\text {kek }}$ & $025^{* k}$ & $0.06^{* * *}$ & 4.04 \\
\hline JNA1/G-4 & 0.05 & -028 & -0.01 & 0.70 & $0.04^{* * k}$ & 499 & $045^{* *}$ & $-0.06^{* * k}$ & $036^{* k *}$ & $-0.07^{* * k}$ & 263 & $0.19^{1 \% *}$ & $-058^{20 k}$ & 153 & -533 & $0.2^{2 k k}$ & 0.003 & -0.001 & 1.86 \\
\hline $\mathrm{JNA} 1 / \mathrm{K} 14 \mathrm{C}$ & -128 & -1.12 & 0.82 & 0.70 & $0.04^{* * *}$ & -699 & -0.06 & $025^{* *}$ & $195^{* * *}$ & $-0.06^{\text {*k }}$ & 4.03 & $-0.14^{*}$ & $0.15^{\text {** }}$ & 353 & $3433^{* * k}$ & $-025^{* *}$ & $-0.17^{* k}$ & $-0.04^{* * *}$ & 137 \\
\hline JNA1/9608U & 038 & -0.12 & -1.01 & -0.97 & $0.04^{* * k}$ & 3.01 & $-1.12^{2 * k}$ & $-0.09^{* * *}$ & $-22^{4 * *}$ & $-0.13^{* *}$ & 3.43 & $-0.18^{* *}$ & $-025^{* * *}$ & $-1280^{10 *}$ & 1.17 & $-0.50^{k 6 k}$ & $-034^{* k}$ & $-0.08^{8 * \%}$ & 1.11 \\
\hline JNA1/BVC-1 & 055 & 1.05 & $299 \%$ & $270^{\text {kek }}$ & $0.03^{* *}$ & 1.01 & 0.05 & $0.10^{k * k}$ & $201^{* * *}$ & $-0.09 \%$ & 3.03 & $047^{* * *}$ & $032^{* *}$ & $5.86^{\text {*k }}$ & 1.00 & $056^{\text {*k }}$ & -0.075 & -0.02 & 211 \\
\hline JNA1/GUK-1 & 1.05 & 055 & 1.16 & 136 & $0.3^{* *}$ & 7.01 & -0.10 & $0.03^{* *}$ & $-09^{* * *}$ & -0.02 & 3.63 & $-0.13^{*}$ & $-051^{\text {**k }}$ & 120 & $-1133^{* *}$ & $084^{* k}$ & -0.08 & -0.02 & 212 \\
\hline JNA1/GUK-2 & 055 & 0.72 & 032 & $153^{*}$ & $0.04^{* * *}$ & 432 & $-0.77^{3 * k}$ & $-0.43^{* *}$ & $-0.82^{* \%}$ & $-0.06^{* * *}$ & 293 & -0.10 & $029^{* * *}$ & 0.86 & $7.00^{*}$ & $-235^{* *}$ & $-022^{* * k}$ & $-0.07^{* * *}$ & 158 \\
\hline JNA1/GUK-2-1 & $27^{2 * *}$ & $338^{* *}$ & $216^{* *}$ & $286^{* * *}$ & $0.03^{* *}$ & -31.66 & 0.04 & $028^{* 2 k}$ & $233^{* * *}$ & $-0.11^{* *}$ & 433 & 0.09 & $039^{\% *}$ & $553^{\text {** }}$ & $1150^{2 k k}$ & $1.00^{\mathrm{kok}}$ & 0.01 & 0.004 & 3.64 \\
\hline JNA1/LCA334 & -0.45 & 1.05 & $249^{\% *}$ & 0.69 & $0.04^{* * k}$ & -39.16 & 0.09 & $-0.12^{* *}$ & $-139^{1 \% k}$ & $-0.09 \%$ & 4.03 & $0.4 \%$ & $-0.45^{* k}$ & $-647^{\text {*2* }}$ & $-11.83^{* * *}$ & $0.10^{\mathrm{k} k}$ & 0.10 & $0.3^{*}$ & 394 \\
\hline JNA1/LCA960 & -0.45 & -0.12 & $216^{* * *}$ & 0.86 & $0.03^{* *}$ & $-80.82^{* * k}$ & $051^{\text {** }}$ & $0.03^{* * *}$ & $-1.6^{7 *}$ & $053^{* * *}$ & 253 & -0.001 & $-028^{* k *}$ & -347 & $-7.17^{*}$ & $042^{* k *}$ & -0.09 & -0.03 & 3.86 \\
\hline JNA1/KDC-1-1 & 0212 & 122 & 0.16 & -030 & $0.04^{* * *}$ & 9.01 & $045^{* *}$ & $0.03^{* *}$ & $-0.3^{* * *}$ & $-0.03^{* * *}$ & 3.13 & $-0.19^{* \%}$ & $1.153^{\text {*k }}$ & $-10.14^{* *}$ & $13.17^{\text {wok }}$ & $-0.45^{* *}$ & 0,08 & 0.017 & 263 \\
\hline JNA1/P. Jwala & 022 & -0.62 & $-266^{2 k *}$ & -1.14 & $0.04^{* * *}$ & 434 & $-0.4 \% *$ & $-024^{* \%}$ & $-139^{1 \% k}$ & $-0.07^{* * k}$ & 3.63 & $026^{* * *}$ & $0.05^{\text {** }}$ & $-647^{\text {*2 }}$ & $-14.67^{\text {** }}$ & $-037^{\text {** }}$ & $-0.16^{\mathrm{kk}}$ & $-0.04^{* * * *}$ & 151 \\
\hline JNA1/KA-2 & 138 & 055 & $5.66^{* * *}$ & $5.9^{9 * k}$ & $0.03^{*}$ & 9.68 & -0.11 & $-0.11^{* *}$ & $051^{* *}$ & $0.03^{* * *}$ & 353 & 0.004 & $0.49^{\text {*** }}$ & $-880^{1 \%}$ & $-2800^{\% *}$ & $020^{10 k}$ & -0.074 & -0.02 & 273 \\
\hline JNA1/K1-4D & 138 & 0.72 & $283^{* * *}$ & $26^{2 * k}$ & $0.03^{*}$ & 3.68 & -0.08 & $-0.12^{* *}$ & $-034^{* *}$ & -0.01 & 3.63 & $037^{* * *}$ & $-035^{\text {*k }}$ & $5200^{2 \%}$ & $-1217^{* *}$ & $057^{\text {tok }}$ & -0.07 & -0.02 & 290 \\
\hline JNA1/PSBR & 055 & 1.05 & 1.16 & $1.86^{*}$ & $0.03^{*}$ & $146.01^{\text {*k }}$ & $-037^{* * k}$ & $-0.08^{1 \% k}$ & $-130^{\% *}$ & $0.03^{*}$ & 333 & $-0.52^{\% *}$ & $-055^{\text {*k }}$ & 153 & $-950^{10 \%}$ & $025^{* k}$ & $038^{* k *}$ & $0.09^{\circ \%}$ & 380 \\
\hline JNA1/BVC-37 & $-3.2^{\text {\%* }}$ & $4.12^{\text {\%* }}$ & $-1.84 *$ & $-21^{* * *}$ & $0.04^{*}$ & $4667^{*}$ & $030^{\text {kok }}$ & $0.09^{* * *}$ & $-030^{1 \% k}$ & $0.116^{* *}$ & 433 & $-038^{* * *}$ & $0.09^{\text {*** }}$ & $9.86^{* * *}$ & $733^{*}$ & $-0.77^{\text {k* }}$ & $0.43^{* *}$ & $0.11^{\text {** }}$ & 4.77 \\
\hline ACA1/SKS & -0256 & -0.87 & $395^{* * *}$ & $493^{* * *}$ & $031^{\text {*k }}$ & -285 & $-0.77^{* * k}$ & $-0.06^{* * *}$ & $-1.81^{* *}$ & $-0.022^{*}$ & 213 & $-0.40^{\circ \%}$ & $0.41^{\text {** }}$ & -343 & 421 & $036^{\text {*k }}$ & $-0.16^{* * k}$ & $-0.04^{* * * *}$ & 1.17 \\
\hline ACA1/PantC-1 & $358^{\circ / *}$ & -036 & $4.78^{\text {ik }}$ & $593^{* *}$ & $-0.02^{* * *}$ & $5331^{*}$ & $-021^{*}$ & $-0.18^{* * *}$ & $-1,9^{1 \%}$ & 0.008 & 323 & $-034^{* *}$ & $-026^{* * k}$ & -1.10 & $-1121^{\text {** }}$ & $-0.42^{* * *}$ & -0.07 & -0.02 & 245 \\
\hline $\mathrm{ACA} 1 / \mathrm{G}-4 /$ & 0.08 & 030 & 0.78 & -1.07 & $-0.02^{2 *}$ & -152 & -0.004 & $0.06^{* * *}$ & $0.74^{* * *}$ & $0.05^{* * *}$ & 283 & 0.06 & $0.07^{* * *}$ & $623^{* *}$ & $11.96^{\text {tok }}$ & $-0.41^{* *}$ & 0.06 & 0.01 & 1.08 \\
\hline ACA1/K1-4C & 025 & 044 & -038 & 0.43 & $-0.02^{2 *}$ & 848 & 0.14 & $-0.18^{* *}$ & $-191^{\% *}$ & $-022^{* * k}$ & 213 & $0.18^{* \% *}$ & $-0.9^{9 k k}$ & -1.76 & $-2637^{* * *}$ & $042^{2 * k}$ & $0.15^{* *}$ & $0.0 *$ & 1.17 \\
\hline ACA1/9608U & -0.59 & -0.53 & 028 & 0.77 & $-0.00^{2 * *}$ & -752 & $050^{\mathrm{kek}}$ & $-0.095^{* * *}$ & $0.69^{* * *}$ & $0.10^{\text {** }}$ & 323 & 0.11 & $-1.09^{\text {ik }}$ & $1290^{*}$ & $-3054 \%$ & $120^{\circ k}$ & $0.16^{* *}$ & $0.04 *$ & 132 \\
\hline ACA1/BVC-1 & -0.92 & -036 & $-322^{* *}$ & $-357^{\text {t*k }}$ & $-0.02^{2 * *}$ & -552 & -0.03 & $022^{* *}$ & $-0.09 \%$ & $0.10^{2 \% *}$ & 3.03 & -0.10 & $-0.03^{\text {*ak }}$ & 0.60 & $679^{*}$ & $0592^{\text {** }}$ & 0.04 & 0.01 & 1.48 \\
\hline ACA1/GUK-1 & -0.92 & -0.86 & $4.05^{* * k}$ & $-340^{\circ k k}$ & $-0.02^{* *}$ & -952 & 0.07 & $-0.02^{* *}$ & $03^{9 k k}$ & $-0.04 \%$ & 323 & $-0.19^{\% *}$ & $-0.16^{* k}$ & $-7.10^{k \%}$ & 296 & $-0.58^{\text {*k }}$ & 0.05 & 0.01 & 152 \\
\hline ACA1/GUK-2 & -0.92 & -120 & -038 & $-1.73^{*}$ & $-0.02^{* * *}$ & -0.85 & -0.02 & $0.03^{* * *}$ & $0.70^{\circ k}$ & $-0.08^{* * *}$ & 3.03 & -0.11 & $-1.16^{\text {*k }}$ & $-9.43^{* *}$ & $20.79 \%$ & $1.73^{* *}$ & 0.06 & 0.02 & 1.47 \\
\hline ACA1/GUK-2-1 & 025 & -0.03 & $-655^{* k}$ & $-640^{\circ / 2 k}$ & $-0.02^{2 * *}$ & 11.81 & -0.04 & $-0.19^{1 \% k}$ & $-0.41^{\% *}$ & $0.17^{* * *}$ & 323 & $-0.43^{* *}$ & $-126^{\text {6*k }}$ & $4.76^{*}$ & $-21.71^{\text {** }}$ & $-022^{1 \% *}$ & -0.06 & -0.02 & 258 \\
\hline ACA1/LCA334 & 0.08 & 1.14 & $3.78^{\text {*k }}$ & $393^{* *}$ & $-0.02^{* *}$ & $4331^{*}$ & $-026^{*}$ & $0.04^{* * *}$ & $045^{* *}$ & $0.032^{* \%}$ & 333 & $-0.19^{* *}$ & $201^{\text {** }}$ & $82^{* *}$ & 346 & $-024^{* *}$ & $-0.18^{2 k k}$ & $-0.05^{* * *}$ & 241 \\
\hline ACA1/LCA960 & $158^{*}$ & $347^{* *}$ & 095 & $210^{\text {kek }}$ & $-0.02^{2 * *}$ & $55.64^{* \% *}$ & $-03^{* *}$ & $0.02^{* * *}$ & $0.66^{* * k}$ & $-026^{* k}$ & 245 & $0.18^{2 * *}$ & $0.67^{\text {*** }}$ & 024 & 263 & $-022^{\text {ki }}$ & 0.04 & 0.01 & 325 \\
\hline ACA1/KDC-1-1 & -026 & 0.80 & 1.45 & $243^{* * *}$ & $-0.02^{* * *}$ & -1552 & $-029 \%$ & $0.09^{1 * k}$ & $-0.00^{1 *}$ & 0.015 & 295 & 0.08 & $-039^{9 * k}$ & $660^{\circ \%}$ & $11.96^{\text {tok }}$ & $-0.92^{* *}$ & -0.07 & -0.01 & 1.41 \\
\hline ACA1/P. Jwala & -026 & -0.03 & $1.2^{*}$ & 0.10 & $-0.02^{2 * *}$ & -219 & $027^{* * *}$ & $0.04^{* * *}$ & $1.01^{* * *}$ & 0.012 & 335 & 0.01 & $061^{*}$ & $524^{* *}$ & $14.13^{\text {*k }}$ & $-055^{* *}$ & 0.11 & 0.03 & 12 \\
\hline ACA1/KA-2 & -0.59 & -0.86 & $-600^{* * k}$ & $-657^{\text {*k }}$ & $-0.02^{2 *}$ & -1286 & 0.18 & $0.02^{2 *}$ & $031^{* *}$ & $-0.025^{*}$ & 275 & $032^{2 * *}$ & $0.04 *$ & 290 & $879 \%$ & $0.19^{\prime k}$ & 0.06 & 0.01 & 213 \\
\hline ACA1/K1-4D & -059 & -0.70 & $1.618^{*}$ & $193^{* * k}$ & $-0.02^{* *}$ & -1285 & -0.02 & $0.04^{* * k}$ & $-0.08^{* * *}$ & $0.035^{* *}$ & 3.05 & $0.17^{*}$ & $021^{\text {** }}$ & $-7.10^{k \%}$ & $7.13^{*}$ & $-035^{* *}$ & -0.01 & -0.003 & 213 \\
\hline ACA1/PSBR & -0.92 & -0.86 & 0.45 & -0.90 & $-0.02^{2 * *}$ & $-74,02^{\text {\%* }}$ & $021^{*}$ & $0.03^{* * *}$ & $0.74^{* * *}$ & $0.04^{* * *}$ & 295 & $036^{* * *}$ & $051^{\text {** }}$ & -1.77 & 1.80 & $-020^{k * k}$ & $-020 \%$ & $-0.00^{* * *}$ & 1.63 \\
\hline ACA1/BVC-37 & 0.41 & 0.47 & 095 & 1.10 & $-0.02^{2 * *}$ & -2735 & $052^{2 *}$ & $0.14^{* * *}$ & $036^{\text {**k }}$ & $0.112^{\text {** }}$ & 325 & $02^{9 \% k}$ & $054^{* *}$ & $-6431^{* * *}$ & 1.63 & $-022^{* *}$ & 005 & 0.01 & 3.05 \\
\hline
\end{tabular}

$*$, **Significant at $\mathrm{p}=0.05$ and 0.01 , respectively. 
Table 6. (contd.)

\begin{tabular}{|c|c|c|c|c|c|c|c|c|c|c|c|c|c|c|c|c|c|c|c|}
\hline Crosses & DFI & DFF & DFR & DFH & NOP & NFP & FFW & DFW & $\mathrm{FL}$ & $\mathrm{FD}$ & PL & PIL & $\mathrm{NPB}$ & PH & NS & SW & FFWP & DFWP & NPY \\
\hline ACA2/SKS & 0.04 & $331^{\text {** }}$ & $3.89 \%$ & $1.87^{*}$ & $031^{\text {** }}$ & -0.82 & 0.18 & $-024^{* * *}$ & $-0.9^{96 \%}$ & -0.02 & 225 & $057^{\text {to }}$ & $034^{* * *}$ & $-9.43^{* * k}$ & $-15.80^{\text {kek }}$ & $-0.97^{* * *}$ & 0.07 & 0.017 & 190 \\
\hline ACA2/PantC-1 & 037 & $181^{*}$ & $3.73^{* * *}$ & $43^{7 * *}$ & $-0.02^{* *}$ & 1283 & $-0.42 * \%$ & $0.05^{* *}$ & $0,49^{2 *}$ & -0.02 & 3.15 & $039 \%$ & $-053^{* *}$ & 090 & $671^{*}$ & $0.9^{1 \% *}$ & $-0.18^{* *}$ & $-0.05^{* * *}$ & 243 \\
\hline $\mathrm{ACA} 2 / \mathrm{G}-4$ & -0.13 & -0.02 & -0.78 & 037 & $-0.02^{* *}$ & 651 & $-0.4 * *$ & -0.002 & $-1.10^{0 \%}$ & $0.03^{*}$ & 265 & $-025^{* *}$ & $051^{* *}$ & $-7.76^{* \% k}$ & $-66^{*}$ & $-0.01^{* *}$ & -0.06 & -0.01 & 1.04 \\
\hline $\mathrm{ACA} 2 / \mathrm{K} 1-4 \mathrm{C}$ & 1.04 & 0.65 & -0.44 & -1.13 & $-0.02^{* *}$ & -1.49 & -0.08 & $-0.07^{1 * k}$ & $-0.04^{*}$ & $031^{* *}$ & 325 & -0.04 & $054^{* * *}$ & -1.77 & $-799^{* k k}$ & $-0.16^{\text {*** }}$ & 0.02 & 0.00 & 1.11 \\
\hline $\mathrm{ACA} 2 / 9608 \mathrm{U}$ & 021 & 0.65 & 0.73 & 021 & $-0.02^{* * *}$ & 451 & $0.62 *$ & $0.19^{* * k}$ & $159^{1 \% *}$ & $0.03^{\text {** }}$ & 265 & 0.08 & $134^{* * *}$ & -0.10 & $2937^{\text {*** }}$ & $-0.70^{02 \%}$ & $0.18^{\text {*** }}$ & $0.04^{* * *}$ & 159 \\
\hline ACA2/BVC-1 & 037 & -0.69 & 023 & 0.87 & $-0.02^{* * *}$ & 451 & -0.02 & $-031^{\text {*k }}$ & $-193^{* * *}$ & -0.01 & 265 & $-037^{* * *}$ & $-029 \%$ & $-643^{* * k}$ & $-7.79^{9 k k}$ & $-1.16^{* * *}$ & 0.04 & 0.01 & 1.69 \\
\hline ACA2/GUK-1 & -0.13 & 031 & $289^{\circ \%}$ & $204^{* * *}$ & $-0.02^{* * *}$ & 251 & 0.03 & -0.007 & $056^{* *}$ & $0.06^{\text {tok }}$ & 205 & $032^{2 *}$ & $0.68^{\% *}$ & $590^{10 *}$ & $837^{* * *}$ & $-026^{* * *}$ & 0.03 & 0.01 & 1.72 \\
\hline ACA2/GUK-2 & 037 & 0.48 & 0.06 & 021 & $-0.02^{2 * *}$ & 5.18 & $0.80^{\circ \%}$ & $0.40^{1 \% k}$ & $0.12^{2 * *}$ & $0.14^{\% *}$ & 3.15 & $021^{\text {** }}$ & $0.87^{* * *}$ & $857^{* * *}$ & $-27.79^{10 k}$ & $0.63^{* * *}$ & $024^{* *}$ & $0.06^{\text {*k }}$ & 212 \\
\hline ACA2/GUK-2-1 & $-296^{* *}$ & $-335^{* *}$ & $439 \%$ & $354^{* *}$ & $-0.02^{* * *}$ & 19.84 & -0.001 & $-0.09^{\text {kek }}$ & $-1.93^{* * *}$ & $-0.05^{* *}$ & 3.05 & $035^{* *}$ & $0.87 \%$ & -0.77 & $1021^{* *}$ & $-0.71^{* *}$ & 0.05 & 0.01 & 3.07 \\
\hline ACA2//LCA334 & 037 & $-219^{*}$ & $-627^{* * k}$ & $4.6^{* * k}$ & $-0.02^{* *}$ & 4.16 & 0.17 & $0.08^{* * *}$ & $0.95^{* *}$ & $0.06^{\text {tok }}$ & 325 & $-029 \%$ & $-156^{* *}$ & -1.77 & $837^{* * k}$ & $0.14^{* * *}$ & 0.08 & 0,02 & 322 \\
\hline ACA2/LCA960 & -1.13 & $-335^{* *}$ & $-3.11^{1 * *}$ & $-296^{* * k}$ & $-0.02^{* *}$ & 25.18 & -020 & $-0.057^{\text {k* }}$ & $1.01^{\text {** }}$ & $-027^{* * *}$ & 265 & $-0.18^{* * *}$ & $-039 \%$ & 324 & 454 & $-0.14^{* *}$ & 0.06 & 0.02 & 354 \\
\hline ACA2/KDC-1-1 & 0.04 & $-202^{*}$ & $-1.61^{*}$ & $-21^{* * *}$ & $-0.02^{* *}$ & 651 & -0.16 & $-0.12^{* * *}$ & $048^{* * *}$ & 0.02 & 3.05 & 0.11 & $-0.76^{* *}$ & 357 & $-25.13^{* * *}$ & $137^{7 * *}$ & -0.003 & 0.00 & 1.79 \\
\hline ACA2/P. Jwala & 0.04 & 0.65 & 1.06 & 1.04 & $-0.02^{* * *}$ & -216 & 0.16 & $020^{1 \% k}$ & $038^{* * *}$ & $0.06^{\text {*2* }}$ & 275 & $-027^{* * *}$ & $-0.66^{* * \%}$ & 124 & 054 & $093^{* *}$ & 0.05 & 0.02 & 132 \\
\hline $\mathrm{ACA} 2 / \mathrm{KA}-2$ & -0.79 & 031 & 039 & 0.87 & $-0.02^{2 * *}$ & 3.18 & -0.07 & $0.09^{* * *}$ & $-0.82 * *$ & -0.004 & 215 & $-033^{* *}$ & $-053^{* * *}$ & $590^{1 \% *}$ & $1921^{\text {*** }}$ & $-03^{9 * *}$ & 0.01 & 0.002 & 227 \\
\hline ACA2/K1-4D & -0.79 & -0.02 & $44^{* * *}$ & $4.6^{3 * *}$ & $-0.02^{2 * *}$ & 9.18 & 0.10 & $0.08^{\text {*** }}$ & $043^{* *}$ & $-0.024^{*}$ & 235 & $-055^{* *}$ & $0.14 * *$ & 190 & 5.04 & $-022^{* *}$ & 0.08 & 0.02 & 259 \\
\hline ACA2/PSBR & 037 & -0.19 & $-1.61^{*}$ & -0.96 & $-0.02^{* * *}$ & $-71.99 \%$ & 0.15 & $0.06^{* * *}$ & $05^{\text {;** }}$ & $-0.07^{* * *}$ & 3.05 & $0.16^{*}$ & $0.04^{* * *}$ & 024 & $7.71^{\text {*** }}$ & $-0.05^{* * *}$ & $-0.18^{* * *}$ & $-0.05^{* *}$ & 1.89 \\
\hline ACA2/BVC-37 & $27^{* * *}$ & $3.6^{* *}$ & 0.89 & 1.04 & $-0.02^{* * *}$ & -1932 & $-0.8^{2 * *}$ & $-023^{* * k}$ & $-0.06^{* 6 \%}$ & $-023^{* * *}$ & 285 & 0.08 & $-0.6^{* * *}$ & -3.43 & $-896^{* * k}$ & $1.00^{1 \% *}$ & $-0.47^{7 * *}$ & $-0.12^{* * *}$ & 210 \\
\hline SED & 0.76 & 090 & 0.75 & 0.71 & 0.00 & 20.10 & 0.10 & 0.004 & 0.02 & 0.01 & 0.05 & 0.06 & 0.00 & 190 & 277 & 0.00 & 0.06 & 0.01 & 0.12 \\
\hline CD $5 \%$ & 153 & 1.80 & 150 & 142 & 0.00 & 4037 & 020 & 0.01 & 0.03 & 0.02 & 0.09 & 0.13 & 0.00 & 381 & 556 & 0.00 & 0.11 & 0.03 & 025 \\
\hline CD $1 \%$ & 204 & 240 & 200 & 190 & 0.00 & 53.82 & 026 & 0.01 & 0.05 & 0.03 & 0.12 & 0.17 & 0.00 & 5.08 & 7.41 & 0.00 & 0.15 & 0.04 & 033 \\
\hline
\end{tabular}

$\mathrm{DFI}=$ Days to first flower initiation, $\mathrm{DFF}=$ Days to fifty per cent flowering, DFR $=$ Days to first fruit ripening, $\mathrm{DFH}=$ Days to first harvest, NOP $=$ Number of pickings, NFP $=$ Number of fruits plant ${ }^{-1}, \mathrm{FFW}=$ Fresh fruit weight fruit ${ }^{-1}(\mathrm{~g}), \quad \mathrm{DFW}=$ Dry fruit weight fruit $^{-1}(\mathrm{~g}), \mathrm{FL}=$ Fruit length $(\mathrm{cm}), \quad \mathrm{FD}=$ Fruit diameter $(\mathrm{cm})$, PL $=$ Pedicel length $(\mathrm{cm}), \mathrm{PTL}=$ Petiole length $(\mathrm{cm}), \mathrm{NPB}=$ Number of primary branches, $\mathrm{PH}=$ Plant height $(\mathrm{cm})$, $\mathrm{NS}=$ Number of seeds fruit ${ }^{-1}, \mathrm{TSW}=1000$ seed weight $(\mathrm{g}), \mathrm{FFWP}=$ Fresh fruit weight plant $^{-1}(\mathrm{~kg}), \mathrm{DFWP}=$ Dry fruit weight plant $^{-1}(\mathrm{~kg}), \mathrm{NPY}=$ Net plot yield $(\mathrm{kg})$.

\section{Conclusion}

It has been concluded that the magnitude of heterobeltiosis was high $(20 \%>)$ with majority of the crosses for most of the traits. Hence, heterosis breeding is favoured as suggested by Patel et al., (2001) in chilli. JNA1/BVC-37 (48.47\%), JNA1/PantC1 (25.76\%) and JNA1/LCA334 (25.33\%) recorded more than 25\% heterosis over best standard check of non male sterility based hybrid. However, F1 hybrids, JNA1/PanC1 (152.63\%), JNA1/LCA334 (370.49\%) and JNA1/PSBR (185.71\%) registered more than 150\% heterobeltiosis, while, three newly developed CMS based hybrids viz., JNA1/LCA960 (69.05\%), JNA1/GUK2-1 (50.85\%) and ACA2/LCA960 (57.17\%) were able to yield more than $50 \%$ heterobeltiosis. Therefore, efforts should be concentrated for the development of male sterility based hybrids using cytoplasmic male sterile lines to minimize the cost of $F_{1}$ seeds and quality seed production in chilli. Cross JNA1/BVC-37 gave the highest yield along with highly significant sca effects thus proved as potential hybrids and could be considered for commercial exploitation of hybrid vigour in chilli (Capsicum annuum L.) after confirmation with multilocational testing.

\section{References}

Allard, R.W. (1960). Principles of Plant Breeding Ist Edn. London, John Wiley and Sons, Inc.

Dash, S. S., Kumar, S., \& Singh, J. N. (2001). Cytomorphological characterization of a nuclear male sterile line of chilli pepper (Capsicum annuum L.). Cytologia, 66(4), 365-371. http://dx.doi.org/10.1508/cytologia.66.365

Fonseca, S., \& Patterson, F. L. (1968). Hybrid vigour in a seven parent diallel cross in common wheat (Triticum aestivum L.). Crop Science, 8, 85-88. http://dx.doi.org/10.2135/cropsci1968.0011183X000800010025x 
Joshi, S., Thakur, P. C., \& Verma, T. S. (1995). Hybrid vigour in bed shaped paparika (Capsicum annuum L). Vegetable Science, 22(2), 105-108.

Kempthorne, O. (1957). An introduction to genetic statistics, New York, John Willey and Sons. pp. 486-211.

Kim, D. H., \& Kim, B. D. (2005). Development of SCAR markers for early identification of cytoplasmic male sterility genotype in chilli pepper (Capsicum annuum L.). Molecular Cells, 20, 416-422.

Kumar, S., Singh, V., Singh, M., Rai, S. K., Kumar S., \& Rai, M. (2007). Genetics and distribution of fertility restoration associated RAPD markers in pepper (Capsicum annuum L.). Scientia Horticulturae, 111, 197-202. http://dx.doi.org/10.1016/j.scienta.2006.10.021

Lee, J., Yoon, J. B., \& Park, H. G. (2008a). A CAPS Marker associated with the partial restoration of cytoplasmic male sterility in chilli pepper (Capsicum annuum L). Molecular Breeding, 21, 95-104. http://dx.doi.org/10.1007/s11032-007-9111-0

Lee, J., Yoon, J. B., \& Park, H. G. (2008b). Linkage analysis between the partial restoration ( $p r)$ and the restorer of fertility $(R f)$ loci in pepper cytoplasmic male sterility. Theory and Applied Genetics, 7, 383-389. http://dx.doi.org/10.1007/s00122-008-0782-7

Parvez, S. (2006). Recent advances in understanding genetic basis of heterosis in rice (Oriza sativa L.). Revista UDO Agricola, 6(1), 1-10.

Patel, J. A., Shukla M. R., Doshi K. M., Patel S. B., \& Patel, S. A. (1997). Hybrid vigour of quantitative traits in chilli (Capsicum annuum L.). Vegetable Science, 24(2), 107-110.

Patel, J. A., Patel, M. J., Patel, A. D., Acharya, R. R., \& Bhalala, M. K. (2001). Hybrid vigour of quantitative traits in chilli (Capsicum annuum L.). Vegetable Science, 28(2), 130-132.

Shifriss, C. (1997). Male sterility in pepper (Capsicum annuum L.). Euphytica, 93, 83-88. http://dx.doi.org/10.1023/A:1002947907046

Turner, J. H. (1953). A study of heterosis in upland cotton, combining ability and inbreeding effects. Agronomy Journal, 45, 487-490. http://dx.doi.org/10.2134/agronj1953.00021962004500100008x

Wang, L. H., Zhang, B. X., Lefebvre, V., Huang, S. W., Daubeze, A. M., \& Palloix, A. (2004). QTL analysis of fertility restoration in cytoplasmic male sterile pepper. Theory and Applied Genetics, 109, 1058-1063. http://dx.doi.org/10.1007/s00122-004-1715-8

Zhang, B. X., Huang, S. W., Yang, G. M., \& Guo, J. Z. (2000). Two RAPD markers linked to a major fertility restorer gene in pepper. Euphytica, 113, 155-161. http://dx.doi.org/10.1023/A:1003945723196 